\title{
Generalized Implicit Functions For Computer Graphics
}

\author{
Stan Sclaroff and Alex Pentland \\ Vision and Modeling Group \\ The Media Laboratory \\ Massachusetts Institute of Technology \\ Cambridge, MA 02139
}

\begin{abstract}
We describe a method of generalizing implicit functions by use of modal deformations and displacement maps. Modal deformations, also known as free vibration modes, are used to describe the overall shape of a solid, while displacement maps provide local and fine surface detail by offsetting the surface of the solid along its surface normals. The advantage of this approach to geometric description is that collision detection and dynamic simulation become simple and inexpensive even for complex shapes. In addition, we outline an efficient method for fitting such models to three dimensional point data.
\end{abstract}

CR Categorles: 1.3.5 [Computer Graphics]: Computational Geometry and Object Modeling; I.3.7 [Computer Graphics]: Graphics and Realism.

Additional Keywords: Collision Detection, Implicit Surfaces, Simulation, Dynamics, Deformations, Solid Modeling, Computer Modeling.

\section{Introduction}

In many graphics applications, and especially in physical simulations, the ability to efficiently detect and characterize collisions and intersections is essential. Unfortunately, the polygon and spline representations normally employed in computer graphics are ill suited to this task. When using a polygon representation, for instance, the computational complexity of collision detection is $O(n m)$ operations, where $n$ is the number of polygons and $m$ is the number of points to be considered after pruning via bounding box considerations [8]. As a consequence, collision detection is one of the most costly operations in many graphics applications [6], despite significant efforts to optimize algorithms for collision and intersection detection $[1 ; 8]$

In contrast, one can perform collision detection relatively efficiently when employing an implicit function representation (e.g., spheres, swept solids, deformable superquadrics (3]) by making use of their inside-outside function. In each case, the computational complexity of this type of collision checking is only $O(m)$ rather than $O(n m)$ [9]. A more subtle but perhaps equally important advantage of this approach is that the collision surface may often be characterized analytically [7; 12], allowing more accurate simulation of multibody collisions.

Permission to copy without fee all or part of this material is granted provided that the copies are not made or distributed for direct commercial advantage, the ACM copyright notice and the title of the publication and its date appear. and notice is given that copying is by permission of the Association for Computing Machinery. To copy otherwise, or to republish, requires a fee and/or specific permission.
Unfortunately, implicit function representations have not been sufficiently expressive for general use. The contribution of this paper will be to show how implicit function representations may be generalized to allow fast collision detection for more general shapes, and to outline an efficient technique for fitting these generalized implicit functions to three dimensional point data.

\section{Generalized Implicit Functions}

An implicit function representation defines a surface as a level set of a function $f$, most commonly the set of points for which $f(\mathbf{x})=0$. For instance, the inside-outside function we use for superquadric ellipsoids, before rotation, translation or deformation, is:

$$
f(\mathbf{x})=\left[\left(x^{2 / \epsilon_{2}}+y^{2 / \epsilon_{2}}\right)^{\epsilon_{2} / \epsilon_{1}}+z^{2 / \epsilon_{1}}\right]^{\epsilon_{1} / 2}-1 .
$$

In practice we have found this better behaved than the standard superquadric inside-outside function, as it is more similar to the a normal $\mathbf{L}_{2}$ distance metric.

A solid defined in this way can be easily positioned and oriented, by transforming the implicit function:

$$
\hat{\mathbf{x}}=\mathbf{M x}+\mathbf{b}
$$

where $\mathbf{M}$ is a rotation matrix, and $\mathbf{b}$ is a translation vector. Similarly, the implicit function's positioned and oriented insideoutside function becomes:

$$
f(\mathbf{x})=f\left(\mathbf{M}^{-1}(\hat{\mathbf{x}}-\mathbf{b})\right) .
$$

To detect a collision between a point $x=(x, y, z)$ and the volume bounded by this surface, one simply substitutes the coordinates of $\mathbf{x}$ into the function $f$. If the result is negative, then the point is inside the surface and a collision has occurred. Generalizations of this basic operation may be used to find line-surface intersections or surface-surface intersections [12].

\subsection{Deformations}

As in Barr $[2 ; 3]$, this basic set of functions can be generalized further by defining an appropriate set of global deformations $\mathcal{D}$ with parameters $\mathbf{u}$. For particular values of $\mathbf{u}$ the new deformed surface is defined using a deformation matrix $\mathcal{D}_{\mathbf{u}}$ :

$$
\hat{\mathbf{x}}=\mathbf{M} \mathcal{D}_{\mathbf{u}} \mathbf{x}+\mathbf{b}
$$

where $\hat{\mathbf{x}}$ is the position vector after rotation, deformation, and translation. Similarly, the inside-outside function becomes

$$
f(\mathbf{x})=f\left(\mathcal{D}_{\mathbf{u}}^{-1} \mathbf{M}^{-1}(\hat{\mathbf{x}}-\mathbf{b})\right) \text {. }
$$

This inside-outside function is valid as long as the inverse deformation $\mathcal{D}_{\mathfrak{u}}^{-1}$ exists. Thus by selecting a set of deformations that can be easily inverted, we can greatly expand the class of shapes that can be described using an implicit function representation. 

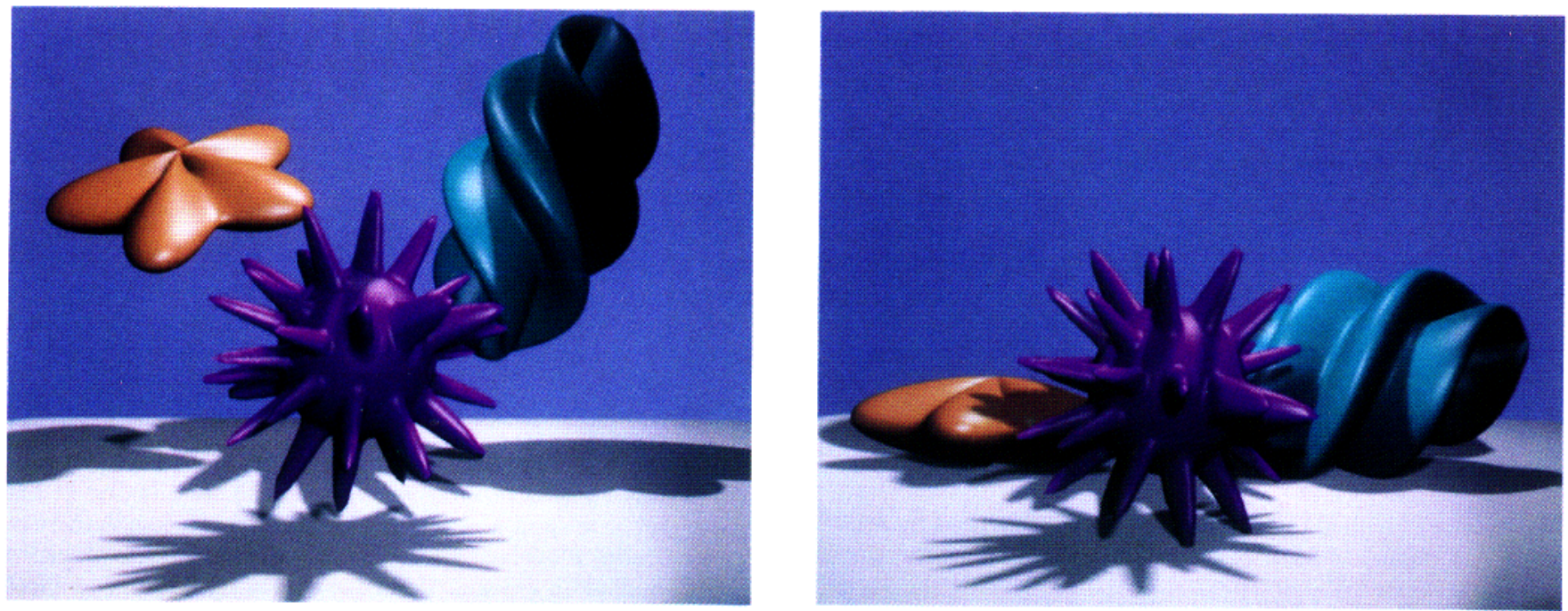

Figure 1: Two frames from a physically-based animation in which seashell-like shapes drop through water and come to rest.

In the Thing World modeling system [ 11$]$ deformations are described by a $3 \times 3$ deformation matrix $\mathcal{D}_{\mathbf{u}}$, referred to as the modal deformation matrix, whose entries are polynomials which mimic the free vibration modes found in real objects. As a consequence, the linear superposition of these deformation polynomials allows accurate description of the dynamic, non-rigid behavior of real objects.

For many computer graphics applications, it is sufficient to pick the following polynomials to be the entries of $\mathcal{D}_{\mathbf{u}}$ :

$$
\begin{aligned}
& d_{00}=u_{6}+y u_{12}+z u_{15}-\left(u_{13}+u_{16}\right) \operatorname{sgn}(x)-u_{14}-u_{17}, \\
& d_{01}=u_{11}+2 z\left(u_{13}+\operatorname{sgn}(x) u_{14}\right), \\
& d_{02}=u_{10}+2 z\left(u_{16}+\operatorname{sgn}(x) u_{17}\right), \\
& d_{10}=u_{11}+2 x\left(u_{19}+\operatorname{sgn}(y) u_{20}\right), \\
& d_{11}=u_{7}+x u_{18}+z u_{21}-\left(u_{19}+u_{22}\right) \operatorname{sgn}(y)-u_{20}-u_{23}, \\
& d_{12}=u_{9}+2 z\left(u_{22}+\operatorname{sgn}(y) u_{23}\right), \\
& d_{20}=u_{10}+2 x\left(u_{25}+\operatorname{sgn}(z) u_{26}\right), \\
& d_{21}=u_{9}+2 y\left(u_{28}+\operatorname{sgn}(z) u_{29}\right), \\
& d_{22}=u_{8}+x u_{24}+y u_{27}-\left(u_{25}+u_{28}\right) \operatorname{sgn}(z)-u_{26}-u_{29} .
\end{aligned}
$$

The parameters $u_{i}$ are the amplitudes of the various free vibration modes, and have simple, intuitive meanings. The lowest frequency modes are the rigid-body modes of translation $\left(u_{0}\right.$ through $u_{2}$ ) and rotation $\left(u_{3}\right.$ through $\left.u_{5}\right)$ which specify the values of $\mathbf{b}$ and $M$. The next-lowest frequency modes are smooth, whole-body deformations that leave the center of mass and rotation fixed: $u_{6}$ through $u_{8}$ control the $\mathrm{x}, \mathrm{y}$, and $\mathrm{z}$ radii, $u_{9}$ through $u_{11}$ are shears about the $\mathrm{x}, \mathrm{y}$, and $\mathrm{z}$ axes, and $u_{12+3 j}$ through $u_{12+3 j+2}$ are tapering, bending, and pinching around the $j^{\text {th }}$ pairwise combination of the $x, y$, and $z$ axes. Note that because the rigid body modes are calculated in the object's coordinate system, they must be rotated to global coordinates before being integrated with the remainder of any dynamic simulation.

\subsection{Displacement Maps}

The class of implicit functions can be generalized still further by defining the surface as the set of points for which $f(\mathbf{x})=d$ for some displacement function $d(\eta, \omega)$, where $\eta, \omega$ are the point's coordinates in the surface's two-dimensional parametric space. We define $\tilde{\mathbf{x}}(\eta, \omega)$ to be the displaced surface point before rotation, deformation or translation:

$$
\tilde{\mathbf{x}}=\mathbf{x}+d \mathbf{n}
$$

This will have the effect of displacing the surface along the surface normal in the original undeformed space before applying the deformation $\mathcal{D}_{\mathbf{u}}$.

In the ThingWorld system displacement maps are stored as twodimensional floating point image arrays. In our examples, the displacement map function $d(\eta, \omega)$ is defined by bilinear interpolation between point samples. If a smoother interpolation is desired, standard spline or pyramid-based multiresolution methods [4] can be employed.

Given a point $\mathbf{x}(\eta, \omega)$ and normal $\mathbf{n}(\eta, \omega)$ on the undeformed implicit surface, deformation transform $\mathcal{D}_{\mathbf{u}}$, and scalar displacement map function $d(\eta, \omega)$, we define $\overline{\mathrm{x}}$ to be the position vector including deformations and displacement map:

$$
\overline{\mathbf{x}}=\mathbf{M} \mathcal{D}_{\mathbf{u}} \tilde{\mathbf{x}}+\mathbf{b}=\mathbf{M} \mathcal{D}_{\mathbf{u}}(\mathbf{x}+d \mathbf{n})+\mathbf{b}
$$

We also need to define $\overline{\mathbf{n}}$ to be the normal vector including deformations and displacement. To find $\overline{\mathbf{n}}$, it is first necessary to find $\tilde{\mathbf{n}}$, the surface normal for the displaced surface before deformation, and then apply deformations to get $\overline{\mathbf{n}}$. The normal $\tilde{\mathbf{n}}(\eta, \omega)$ is derived by taking the cross product of the partial derivatives for the undeformed displaced surface function, $\tilde{\mathbf{x}}(\eta, \omega)$ :

$$
\frac{\partial \tilde{\mathbf{x}}}{\partial \eta}=\frac{\partial \mathbf{x}}{\partial \eta}+d \frac{\partial \mathbf{n}}{\partial \eta}+\mathbf{n} \frac{\partial d}{\partial \eta}
$$

and

$$
\frac{\partial \tilde{\mathbf{x}}}{\partial \omega}=\frac{\partial \mathbf{x}}{\partial \omega}+d \frac{\partial \mathbf{n}}{\partial \omega}+\mathbf{n} \frac{\partial d}{\partial \omega} .
$$

In our current implementation, the partials are calculated by finite differences.

The inside-outside function associated with Equation 7 is then:

$$
f(\mathbf{x})=f\left(\mathcal{D}_{\mathbf{u}}^{-1} \mathbf{M}^{-1}(\tilde{\mathbf{x}}-\mathbf{b})-d \mathbf{n}\right) .
$$

It is difficult to evaluate a displacement mapped and deformed inside-outside function because we cannot know $d$ or $\mathbf{n}$ in Equation 9 before we know $x$, and vice versa. We therefore need a parametric projection function $P(\tilde{\mathbf{x}})=(\eta, \omega)$, which can project an undeformed, displacement-mapped $\tilde{\mathbf{x}}$ point back onto the original, undisplaced implicit surface. These parameters can then be used to determine $\mathbf{n}, \mathbf{x}$, and $d$. The projection should be normal projection since the displacement map displaces surface points along the surface normal. 
For example, the projection function $P(\tilde{\mathbf{x}})=(\eta, \omega)$ for superquadric ellipsoids is computed as follows. We first find $\omega$ by observing:

$$
\frac{\tilde{y}}{\tilde{x}}=\frac{\cos ^{\epsilon_{1}} \eta \sin ^{\epsilon_{2}} \omega}{\cos ^{\epsilon_{1}} \eta \cos ^{\epsilon_{2}} \omega}=\tan ^{\epsilon_{2}} \omega
$$

where $(\tilde{x}, \tilde{y}, \tilde{z})^{T}=\tilde{\mathbf{x}}$ is the undeformed, displaced surface point. From Equation 10, we see that $\omega=\operatorname{atan}^{1 / \epsilon_{2}}(\tilde{y} / \tilde{x})$. The remaining parameter, $\eta$, is determined by either $\eta=\operatorname{atan}^{1 / \epsilon_{1}}\left(\left(\tilde{z} \cos ^{\epsilon_{2}} \omega\right) / \tilde{x}\right)$ or $\eta=\operatorname{atan}^{1 / \epsilon_{1}}\left(\left(\tilde{z} \sin ^{\epsilon_{2}} \omega\right) / \tilde{y}\right)$ depending on whether $\tilde{x}$ or $\tilde{y}$ is larger.

\subsection{An Example}

Figure 1 shows two frames from a physically-based animation in which three seashell-like shapes drop through a viscous medium (e.g., seawater), hit the sea bottom, bump into each other, and then come to rest. The simulations were conducted using the technique of modal dynamics as implemented in the ThingWorld system [11].

The seashell shapes were modeled as superquadric ellipsoids with displacement maps. Each displacement map consisted of a $100 \times 100$ uniformly spaced grid generated by combinations of sines and cosines. The shells were polygonalized for display and simulation purposes - approximately 2300 polygons for the spike seashell, and 576 polygons for the other seashells. Bounding boxes were also computed for the objects. During the simulation, if these bounding boxes crossed, then polygon vertices were plugged into the offending objects' inside/outside functions to test for collisions.

Execution time for the three active objects was 0.05 seconds per time step during the initial frames of the animation (before any contact), and 0.081 seconds per time step during the final few frames, when the three seashells were colliding with the seabed (which is not planar), and the shells were colliding with each other. Subtracting the pre-contact time from the execution time during contact, we find it took approximately 0.031 seconds per time step for contact detection and calculation of the non-rigid dynamics. Contact detection, physical simulation, and geometric updates were computed on a Sun $4 / 330$, with a TAAC board performing rendering.

\section{Fitting 3-D Point Data}

It is useful to be able to fit a generalized implicit function representation to three-dimensional point data, so that objects in the world can be sampled and brought into our computer where they can participate in simulations. To fit point data with a generalized implicit function requires determining both the deformation parameters $\mathbf{u}=\left(u_{1} \ldots u_{m}\right)^{T}$ used in $\mathcal{D}_{\mathbf{u}}$, and a displacement map.

Let us assume that we are given $n$ three-dimensional sensor measurements $\overline{\mathbf{X}}$ (in the global coordinate system) that originate from the surface of a single object:

$$
\overline{\mathbf{X}}=\left[\bar{x}_{1}, \bar{y}_{1}, \bar{z}_{1}, \cdots \bar{x}_{n}, \bar{y}_{n}, \bar{z}_{n}\right]^{T}
$$

We need to determine a mapping between $X$, points on the undeformed surface, and $\overline{\mathbf{X}}$, the sensor measurements that specify the points' target positions after displacement and deformation. To determine this mapping, we first define an ellipsoidal coordinate system by examination of the data's center of mass and central moments of inertia. For a detailed description of this initialization step, see [10]. This ellipsoid will serve as our initial guess of the undeformed implicit surface.

The sensor measurements are then projected onto this ellipsoid by using the projection function, $P(\mathbf{x})$. This projection implicitly defines a correspondence between the undeformed surface points and their desired positions after deformation. When the number of data points is large it is more efficient to project the data onto a predetermined grid of undeformed surface points. Each data point's position is distributed among nearby surface points using a Gaussian weighting [13].

\subsection{Recovering Deformation Modes}

Once point correspondences have been established, we can proceed with fitting. The task will be to deform the original undeformed points, $\mathbf{X}$, to their desired positions, $\overline{\mathbf{X}}$. At the end of this process, we will have recovered the deformed implicit function which best fits the data points.

To begin with, the effect of each of the $m$ deformation parameters $u_{i}$ in $\mathcal{D}_{\mathbf{u}}$ on the position of the undeformed points, $\mathbf{X}$ is calculated. to obtain a $\mathrm{m} \times 3 \mathrm{n}$ matrix $\Phi$ whose $i^{\text {th }}$ column $\phi_{i}$, is:

$$
\phi_{i *}=\left(\frac{\partial x_{1}}{\partial u_{i}}, \frac{\partial y_{1}}{\partial u_{i}}, \frac{\partial z_{1}}{\partial u_{i}}, \ldots \frac{\partial x_{n}}{\partial u_{i}}, \frac{\partial y_{n}}{\partial u_{i}}, \frac{\partial z_{n}}{\partial u_{i}}\right)^{T}
$$

The matrix $\boldsymbol{\Phi}$ can be computed by finite differences - i.e., analytically, or by applying a small amount of each deformation $u_{i}$ and measuring the resulting change in the coordinates of each point. In the ThingWorld modeling system the deformations used are the object's free vibration modes, so that the columns of $\Phi$ define a coordinate transformation that diagonalizes the object's finite element equations. This allows the object's rigid and nonrigid dynamics to be simulated very inexpensively, as described in reference [11].

The matrix $\boldsymbol{\Phi}$ is the Jacobian of $\mathcal{D}_{\mathbf{u}}$ at each point in $\mathbf{X}$, and so may be used in a modified Newton-Raphson iteration to obtain the minimum RMS error estimate of deformation parameters $\mathbf{u}$ as follows:

$$
\mathbf{u}^{k+1}=\boldsymbol{\Phi}^{-1}\left(\overline{\mathbf{X}}-\mathbf{X}^{k}\right)+\mathbf{u}^{k}
$$

where $\mathbf{X}^{k}$ is the projection of the data points on the surface defined by the deformations $\mathbf{u}^{k}$ at iteration $k$, and $\mathbf{u}^{0}=\mathbf{0}, \mathbf{X}^{0}=\mathbf{X}$. We have found that a single iteration is often sufficient to obtain a satisfactory estimate of the deformation parameters $\mathbf{u}$. Because $\boldsymbol{\Phi}$ is usually not square, use of the pseudoinverse $\boldsymbol{\Phi}^{-1}=\left(\boldsymbol{\Phi} \boldsymbol{\Phi}^{T}\right)^{-1} \boldsymbol{\Phi}^{T}$ is required; as ( $\boldsymbol{\Phi} \Phi^{T}$ ) is only an $\mathrm{m} \times \mathrm{m}$ matrix, this calculation is inexpensive.

\subsection{Computing a Displacement Map}

If there are more degrees of freedom in the data points than in the deformation parameters, the deformed model will not generally pass through the data points exactly (i.e., $f\left(\mathcal{D}_{\mathbf{u}}^{-1} \mathbf{M}^{-1}(\overline{\mathbf{x}}-\mathbf{b})\right) \neq 0$ ). A more accurate approximation to the data points can be obtained by incorporating these residual differences into a displacement map. This is done by solving for the displacement map value $d(\eta, \omega)$ that yields $\overline{\mathbf{x}}=\mathbf{M} \mathcal{D}_{\mathbf{u}}(\mathbf{x}+d \mathbf{n})+\mathbf{b}$ for some point $\mathbf{x}$ on the undeformed implicit surface. The final result is a generalized implicit function representation that normally provides an exact fit to the set of initial data points, and provides a smoothly interpolated surface between those points - except in certain degenerate cases, such as when Nyquist criteria are not statisfied.

To compute the displacement map each data point $\overline{\mathbf{x}}$ is subjected to the inverse deformation $\mathcal{D}_{\mathbf{u}}^{-1}$ to obtain $\tilde{\mathbf{x}}$, the point in the undeformed space. Next, we project $\tilde{\mathbf{x}}$ along the surface normal using $P(\tilde{\mathbf{x}})$ to obtain its two-dimensional parametric space coordinate $(\eta, \omega)$. Finally, we compute the undeformed point's normal distance to the undeformed implicit surface by substituting it's coordinates into the surface's inside-outside function:

$$
d(\eta, \omega)=f(\tilde{\mathbf{x}})
$$

In the ThingWorld system displacement maps are represented by a regularly spaced grid in the surface's parametric space. Thus as each point is projected and its displacement determined, the result is spread to nearest grid points by Gaussian weighted averaging. This interpolation method works well when data points are fairly dense, however when there are only a few data points more sophisticated interpolation methods must be used [5]. 

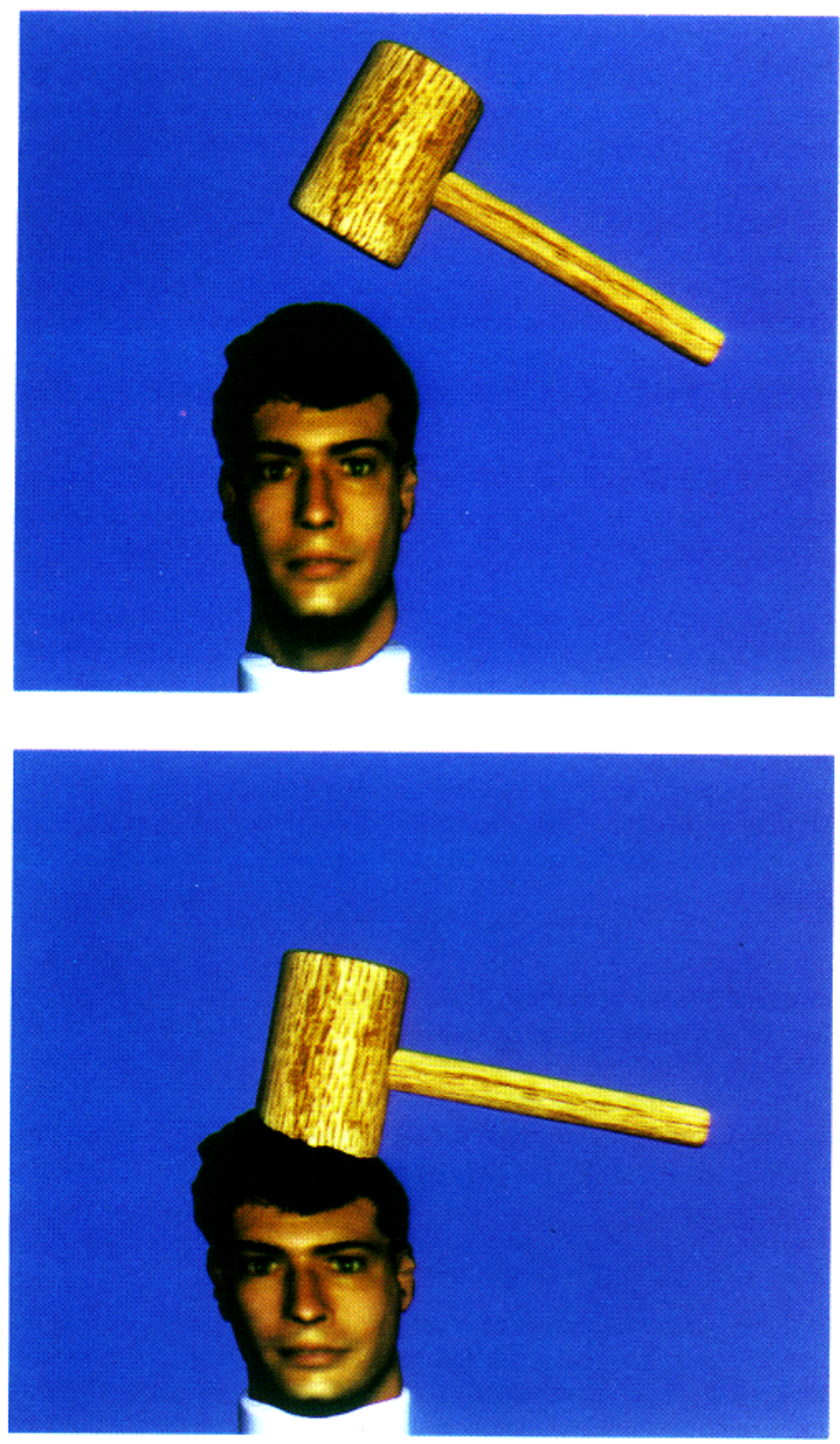

Figure 2: Two frames from a physically-based animation in which a head deforms in response to getting bonked.

\subsection{Example: Modeling a Head}

Figure 2 shows two frames from a physically-based animation in which a jello-like head is struck with a wooden mallet. The head shape was recovered by converting $360^{\circ}$ laser range data of a human head that was scanned by Cyberware at SIGGRAPH' 90 .

The model for the head was recovered with the method described above. It took about 5 seconds on a Sun $4 / 330$ to recover the deformations and displacement map from 2500 data points. A color map had to be computed at higher resolution - about 10,000 data points were used. The color map was computed by projecting the color points onto the surface using $P(\mathbf{x})$ and gaussian interpolation for smoothing.

For the simulation, the head was sampled with 7200 polygons. The other objects had about 600 polygons each. Execution time for the three active objects averaged 0.13 seconds per time step during the collision, with approximately 0.05 seconds per time step attributable to contact detection and calculation of the nonrigid dynamics. The greater execution times for this example are primarily due to the large amount of detail in the head model.

\section{Conclusion}

Pentland and Williams [ 11 1] presented a method for combining implicit function representations with modal dynamics to obtain near-real-time non-rigid dynamic simulations. This paper extends this method by developing a generalized implicit function representation that is sufficiently powerful to describe many of the objects commonly used in computer graphics. The representation can therefore be used to reduce the cost of contact detection and physical simulation in most computer graphics applications. Even degenerate contact geometries, such as vertex-edge intersections, can be handled by techniques such as described in $[3 ; 7 ; 12]$.

Finally, we have presented an efficient method for converting from surface point position data to our generalized implicit function representation. This allows automatic calculation of an implicit function representation from either physical measurements (e.g., from laser rangefinders, or Polhemus measurements) or from vertex position data (e.g., from polygon vertices, or samples from spline surfaces). For a more detailed description of our fitting method, initial model placement, and applications, see [10].

\section{Acknowledgments}

We would like to thank the ThingWorld programming posse: Irfan Essa, Thad Starner, Brad Horowitz, and Martin Friedmann.

\section{References}

[1] D. Baraff. Curved Surfaces and Coherence for Nonpenetrating Rigid Body Simulation. Computer Graphics, 24(4):19-28, 1990.

[2] A. Barr. Global and Local Deformations of Solid Primitives. Computer Graphics, 18(3):21-30, 1984.

[3] A. Barr. Superquadrics and Angle-Preserving Transforms. IEEE Computer Graphics and Applications, 1(1): 1 1-23, 1981.

[4] P. J. Burt and E. H. Adelson. A Multiresolution Spline With Application to Image Mosaics. ACM Transactions on Graphics, 2(4):217-236, 1983.

[5] T. Foley, D. Lane, and G. Nielson. Interpolation of Scattered Data on Closed Surfaces. Computer Aided Geometric Design, 7:303-312, 1990.

[6] J. K. Hahn. Realistic Animation of Rigid Bodies. Computer Graphics, 22(4):299-308, 1988.

[7] D. Kalra and A. H. Barr. Guaranteed Ray Intersections with Implicit Surfaces. Computer Graphics, 23(3):297-306, 1989.

[8] M. Moore and J. Wilhelms. Collision Detection and Response for Computer Animation. Computer Graphics, 22(4):289$298,1988$.

[9] A. Pentland. Computational Complexity Versus Virtual Worlds. Computer Graphics, 24(2): 185-192, 1990.

[10] A. Pentland and S. Sclaroff. Closed-Form Solutions for Physically-Based Shape Modeling and Recognition. IEEE Trans. on Pattern Analysis and Machine Intelligence, 13, to appear in July 1991. Special Issue on Physically-Based Modeling.

[11] A. Pentland and J. Williams. Good Vibrations : Modal Dynamics for Graphics and Animation. Computer Graphics, 23(4):215-222, 1989.

[12] B. von Herzen, A. Barr, and H. Zatz. Geometric Collisions for Time-Dependent Parametric Surfaces. Computer Graphics, 24(4):39-48, 1990.

[13] L. Williams. Performance-Driven Facial Animation. Computer Graphics, 24(4):235-242, 1990. 